\title{
أركون و "نقد العقل الإسلامي" قراءة في "كيف نفهم الإسلام اليوم؟"
}

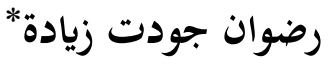

يواصل أركون في كتابه الجديد توضيح ملامح مشروعه في "نقد العقل الإسلامي" الذي كان قد قدمه

لأول مرة عام 1984م، والذي يهدف من ورائه إلى إعادة تقييم نقدي شامل لكل الموروث الإسلامي منذ

ظهور القرآن حتى اليوم مميزا فيه بين أربع مراحل رئيسية:

- مرحلة القرآن والتشكيل الأول للفكر الإسلامي.

- مرحلة العصر الكلاسيكي، الذي يدعوه بعصر العقلانية والازدهار العلمي والحضاري.

- مرحلة العمر السكولاستيكي (المدرسي) التكراري الاجتراري، أو ما يدعى بعصر الانطاط.

- مرحلة النهضة في القرن التاسع عشر حتى الخمسينات من هذا القرن.

ويرو أنه ينبغي أن نضيف مرحلة خامسة يدعوها بّ"الثورة القومية" (عبد الناصر 1952-1970) ف

$$
\text { "الثورة الإسلامية" (1979 حتى اليوم). }
$$

غير أنه لا نخرج بعد قراءة الكتاب على مشروع متبلور، واضح الملامح والقسمات، وإنما - كما هي

عادة أركون في كتبه الأخرى1 - نجد الكتاب عبارة عن مجموعة من الدراسات نشرت سابقا وبعضها لم

$$
\begin{aligned}
& \text { F طبيب أسنان وباحث مهتم بقضايا التجديد في الفكر العربي الإسلامي المعاصر، سورية. } \\
& 1
\end{aligned}
$$

M. Arkoon, Gritique delaraison Islamique, maisonneuve et larose, Paris, 1984.

$$
\text { - إعادة التفكير في الإسلام اليوم، الجزائر، 1991م. }
$$

Penser I'sislamaujoud'fui, Alger, 1991.

$$
\text { - قراءات في القرآن، ط2، تونس، 1991م. }
$$

Lectures ducoran, Tunis, 1991.

$$
\text { - آفاق مشرعة على الإسلام، ط2، باريس، 1993م. }
$$

Ouvertwes sur I'Islam, Paris, 1993.

$$
\text { بالإضافة إلى كتب أخرى متعددة كتبها أركون بالفرنسية، بعض هذه الكتب فقط ترجم إلى العربية، نذكر منها: }
$$


ينشر، رأى مؤلفها أن يضمها بين دفتي كتاب واحد يقوم بترجمته - كما هي العادة أيضا هاشم صالح** الذي يجب ان نعترف له أن تربته شهدت تطورا ملحوظا بحيث أصبحنا نقرأ نصا وكأنه غير مترجم، إلا أنه أثقل كاهلنا بموامش كثيرة مبررة وغير مبررة، وربما شعر هو نفسه بذلك في الدراسات الأخيرة مما يفسر اختفاء الهوامش كليا منها.

يضم الكتاب خمس دراسات مطولة تحمل الأولى عنوان (كيف ندرس الإسلام اليوم؟) وهي تبدأ من مقولة التواصل المستحيل التي يراها بينه وبين المستشرقين الغربيين من جهة والجمهور العربي الإسلامي من

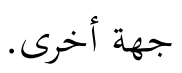

ولكن أبرز ما في دراسته تلك فرضيته التي تقوم على أن يسوع الناصري تكلم وبشر بالآرامية، لكن الأناجيل كتبت باليونانية، ثم انتقلت الكاثوليكية الرومانية إلى اللغة اللاتينية، ثم انتقلت مع لوثر إلى اللغات الأوربية الحديثة، وفي كل مرة كانت تتغير فيها اللغة، كانت تحصل انطلاقة جديدة للشيفرة اللغوية والدلالية والثقافية، أما المسلمون فإفم يكتفون باستغلال الامتياز اللاهوتي لاستمرارية اللغة التي استخدمت لأول مرة في الوحي، وهذا أكبر دليل على أن المشاكل الأكثر عمقا والخاصة بالعلاقات الكائنة بين اللغة والفكر تظل مرمية في ساحة اللامفكر فيه. والنتيجة التي يخلص إليها هي أن العلاقة بين اللغة والدين هي معطى تاريخي ونفسي - ثقافي متغير بالضرورة.

هذه الفرضية التي تحمل في طياتما دلالات خطيرة - مهما حاول المترجم في هامشه حرف الفكرة عن مسارها - تبدأ من تاريخية اللغة والنص، وما يستتبع ذلك من تغيير في لغة النص نفسها على اعتبار أغا محكومة بظروف نشأتها التاريخية والبيئة.

- - ماريخية الفكر العربي الإسلامي، ترجمة هاشم صالح، بيروت؛ مركز الإنماء القومي، ط1، 1986.

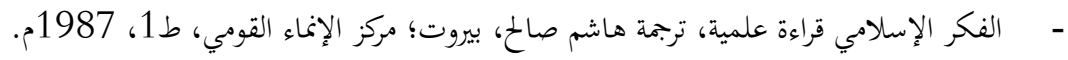

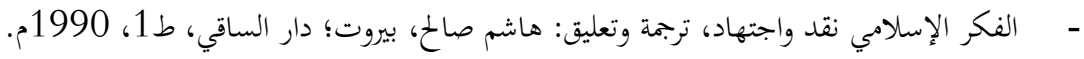

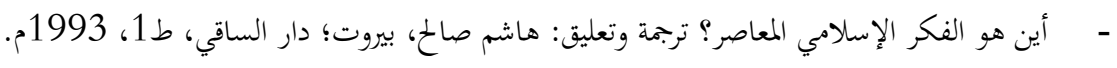
كاتب سوري مقيم في باريس، ترجم العديد من كتب أركون إلى اللغة العربية. 
وبعد ذلك ينتقل ليعدد أهم المراجع في مشروعه لنقد العقل الإسلامي ذاكراكتاب المستشرق الألماني جوزف فان ايس الذي صدر بين عامي 1991-1997م في ستة أجزاء حاملا عنوان (اللاهوت والمجتمع في القرنين الثاني والثالث للهجرة) تاريخ الفكر الديني في بدايات الاسلام. جاعلا إياه حدثا ثقافيا، ومنوها بضرورة ترجمته إلى لغات المجتمعات الاسلامية كافة، ومشروع (انسكلوبيديا القرآن) أو الموسوعة القرآنية، التي صدرت عن الناشر الهولندي (بريل) ويترأس المشروع البرفسورة جين دام ماك أوليف وقد صنفت الموسوعة بحسب ترتيب الحروف الأبجدية لمفاهيم الواردة في القرآن، وسيعالج كل مفهوم قرآني من قبل أحد الاختصاصيين.

أما الدراسة الثانية (خرق الحدود التقليدية، زحزحتها عن مواقعها، بتحاوزها) فهي مخصصة لأعمال المستشرق المعروف كلود كاهين، مما جعل أركون يفرغ ما في جعبته من نقدٍ للمستشرقين - وهو عموما ليس بالشيء القليل - الذين ضخموا من تأثير الإسلام إلى درجة أفم جعلوه - أي الإسلام - يشمل كل شيء ويتدخل في كل شيء، وكأنه أقنوم هائل مهيمن يمكن عن طريقة تفسير كل ما يحدث في العالم الذي انتشر فيه هذا الدين، دون أخذ أي اعتبار للعوامل الانثربولوجية والثقافية والاجتماعية والسياسية، مما جعله مصطلحا غامضا عموميا فضفاضا، قابلا للاستخدام هنا وهناك وفي كل مكان، من أجل الإجابة عن جميع الأسئلة وحل كل المعضلات، إنه المصطلح المهيمن المنتشر في جميع الظروف والأحوال، إنه يستخدم وكأنه

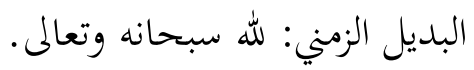

إضافة لذلك فإن الدراسات الاستشراقية تفتقر إلى توظيف أحدث أنواع المناهج والمصطلحات والأدوات العلمية، هذا في حين أها تستخدم بشكل فعال في دراسة التراث الغربي، مما يعكس بشكل صارخ مدى تخلف الدراسات الاستشراقية بالقياس إلى الدراسات الأوربية، والنقطة الأخرى التي يأخذها على المستشرقين هي عدم اهتمامهم بولادة الحركات الشعوبية معتبرين إياها ظاهرة لا تدخل ضمن نطاق اختصاصاقم لأفم يدرسون الماضي، لكن أركون يؤكد لهم أنه لا يمكننا الجزم أبدا بأن هذه الظاهرة لا تؤثر إطلاقا على منهجية الاستشراق أو على الإشكاليات التي يطرحها. لقد حصر الاستشراق نفسه ضمن عقلية ضيقة وضعيفة لا تعترف إلا بالعوامل المادية البحتة في التاريخ، منكرا البعد الروحي بصفته أحد المكونات 
التي تشكل نظام التصور والاعتقاد الخاص بكل مجتمع، فعند دراسة القوى التي لعبت دورا مهما في حسم حركة التاريخ أو التأثير على وجهته يجب ألا غمل العامل الروحي وذلك لأنه حاضر بنسبة ما، والدراسات الاجتماعية الحديثة تؤكد ضرورة عدم إهماله.

فضلا عن ذلك فإن المستشرقين عليهم أن يكفوا عن الادعاء بأنه لا يمكننا الفصل بين العامل الروحي /الزمني، أو بين الدين/الدنيا، أو بين الدين/السياسة في الإسلام ويتبعهم في ذلك كثير من الإسلاميين الذي يعدون مقولة المستشرقين تلك انتصارا لهم غير مدركين أن المستشرقين إنما يقولون ذلك لينتهوا إلى أن المجتمعات المسلمة لن تتمكن من الدخول في عصر الحداثة والتقدم لأن الإسلام دين لا يسمح بذلك. وفي دراسته الثالثة (الإسلام المعاصر أمام تراثه والعولمة) فهي تدور حول (الإسلام والعولمة) حيث يرى أركون أن ظاهرة العولمة تنشر على مستوى الكرة الأرضية بأسرها استرابتيات اكتساح الأسواق الاستهلاكية وتدجينها وإلحاقها بها، ولا تبالي إطلاقا بالمآسي الاجتماعية والفقر المدقع الناتج عن ذلك في دول الجنوب، كما لا تبالي بالتراجعات الفكرية والقمع السياسي والبؤس الثقافي الناتج بالضرورة عن سياسة (التبادل غير المتكافئ) التي تقوم على إقامة علاقات دبلوماسية مح دول الجنوب، وعقد صفقات بتحارية معها من أجل تصدير سلعها الاستهلاكية واستيراد المواد الأولية اللازمة لمصانعها، وهي من جهة ثانية توعز لوسائل إعلامها بفتح أبواقها على سياسات هذه الدول بالذات متهمة إياها بالاستبداد والتعصب الديني والتخلف وعدم احترام حقوق الإنسان، وكل ذلك في الوقت الذي تعترف به رسميا بهذه الأنظمة وتعتبرها بمثابة الشريك المحرم، ومن ثََّّ فهو يرى أن الاحتجاجات العنيفة ضد ظاهرة العولمة ليست محصورة بالمجتمعات الإسلامية وإنما هي مشتركة لدى جميع الشعوب، إذ تمثل رد فعل ضد القوى العمياء والكاسحة للعولمة.

وهذا يشكل برأيه أحد الأسباب الكامنة وراء انتشار ظاهرة الأصولية، اضافة إلى عوامل أخرى كثيرة من مثل تخلي الأنظمة السياسية عن شعوبها لأها مشغولة باحتكار العنف الشرعي (أي القمع) أكثر مما هي ردمي مشغولة باكساب مشروعية ديمقراطية حديثة، كما أها مشغولة بالنهب والسلب، ويضاف إلى ذلك تزايد الهوة الاجتماعية والاقتصادية بين قلة مترفة وأكثرية مسحوقة تئن تحت الفقر المدقع، كل ذلك لعب دورا في خلق المناخ المناسب والمؤهب لانتشار العنف بكل أشكاله. 
إن العولمة قد فرغت مفاهيم من مثل الغرب، الشرق، الإسلام، التنمية، حقوق الإنسان، حقوق الشعوب... من معناها وحولتها إلى قوالب شكلية في استراتيجية السياسات الدولية، لقد تحولت تلك المفاهيم إلى أقنومات ذهنية بتريدية لا معادل لها على أرض الواقع، ولذلك فقدت مصداقيتها في نظر أبناء العالم الثالث الذين طالما خابت آمالهم في التنمية المحسوسة والحقيقية، إغم لم يعودوا يثقون بالشعارات الطنانة أو الرنانة التي لا تطبق ولا تؤتي ثمارها، فمراكز الميمنة الدولية هي التي تخلق هذه الشعارات من أجل تحقيق مآربها الخاصة لا من أجل انتشال الشعوب المنكوبة من الحفرة التي وقعت فيها، حفرة الفقر والحرمان

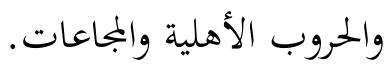

بعد ذلك يأخذ أركون في طرح أسئلة، تعمل الأوضاع العربية والإسلامية الحالية على تغيبها والفقز من فوقها، اسئلة تدور حول النص القرآني وتاريخ إقفال النقاشات الدائرة حوله بتاريخ الطبري، مما جعل تفسيره بمثابة التأسيس التدشيني الأولي للأرثوذكسية الإسلامية، أي للخط الرسمي الذي شاع وهيمن فيما بعد بصفته الحطط الوحيد الصحيح، وما عداه فهرطقة وضلال، وهكذا فإن هناك صيرورة تاريخية معقدة ومليئة بالصراعات، وهي التي أدت في فاية المطاف إلى تشكيل المدونة النصية الرسمية، المغلقة والنهائية بصفتها أمرا واقعا. لكنه يعود فيؤكد ضرورة دراسة الفترة الممتدة من القرن الثالث عشر إلى التاسع عشر التي أُهملت كثيرا بحجة أها عصور انخطاط أو سبات طويل أو عودة إلى الخرافات الشعبية، وينبع اهتمامنا بها من أن المجتمعات العبية الإسلامية الراهنة ناتجة عنها مباشرة، وعليه، فلا يمكن فهم المشاكل الحالية إن لم نفهم تلك الفترة من كل جوانبها.

إن أركون يرى أن العقل العربي الإسلامي قد شهد قطيعتين تاريخيتين الأولى داخلية حين انقطع الفكر الإسلامي ذاته عن المرحلة الكلاسيكية أي القرون الهجرية الستة الأولى من تاريخ الاسلام (حتى وفاة ابن رشد في أواخر القرن الثاني عشر، حيث يعتبر أركون أن مرحلة الانطاط بدأت بعد وفاته)، والقطيعة الأخرى خارجية حين نام الفكر العربي الإسلامي وجمد ولم يعد يعرف ماذا يكصل حوله من اكتشافات علمية وتطورات فلسفية في العالم الغربي، وهكذا تشكلت الحداثة المادية والفكرية خارجه وبمنأى عنه دون أن تتاح له المساهمة فيها، إذ تحول المسلمون إلى مستهلكين فقط، يستهلكون منتجات الحداثة ولا ينتجوغا، فلا 
توجد أي مساهمة فلسفية عربية أو إسلامية في النقاش الدولي الدائر حاليا حول مسألة الحداثة وما بعد الحداثة. ويخلص إلى نتيجة مفادها أن دخول المسلمين التاريخ مشروط إلى حد كبير بتوجهات الفكر الفلسفي التي ستتمخض عنها أزمة العولمة والحداثة.

أما الدراسة الرابعة (إضاءة الماضي لفهم الحاضر والمستقبل) فهي تُعَدُّ بحق امتدادا للدراسة السابقة، حيث تعمل على فضح الخطاب السياسي السائد حاليا في الغرب حول الديمقراطية وحقوق الانسان الذي يقول إنه لن يتعامل مع أنظمة لا تحترم الحد الأدنى من حقوق الانسان ولكنه ظل لا يتعامل فحسب بل يدعم هذه الأنظمة التي تدوس برجليها كل يوم حقوق الإنسان، ومارس أركون أيضا نقده للخطاب الإسلامي السياسي الذي حول الدين إلى أيديولوجيا سياسية أفقدت الدين روحانيته المتعالية ونزلته من تنزيهه المتعالي إلى أرضية الصراعات اليومية والمناورات السياسية المحتومة والمتقلبة، وهذا ما لا يليق بالدين، لكن يبدو هنا أركون متناقضا مع نفسه بعض الشيء إذكيف ينطلق في دراسته الأولى من أن الدين والنص تاريخيان منذ لحظة تأسيسهما وتدشينهما الأولى، لينتهي هنا إلى أن الدين هو منزه ومتعالٍ وخارج عن التاريخ.

مهما يكن مِن أمر فإن أركون ينادي بالتحرير الثاني للبلدان العربية الإسلامية باعتبار أن التحرير الأول وهو التحرير السياسي قد حصل، ويجب أن يتم التحرير الثاني الذي هو التحرير الفكري، وهي دعوة ليست لمرد (إصلاح ديني) وإنما لثورة فكرية حقيقية تذهب إلى أعماق الأشياء وتغير منظورنا جذريا للتراث. إن هذا التحرير الفكري هو الذي سيمكننا من الانخراط في سلك الحضارة الكونية، ووحدها

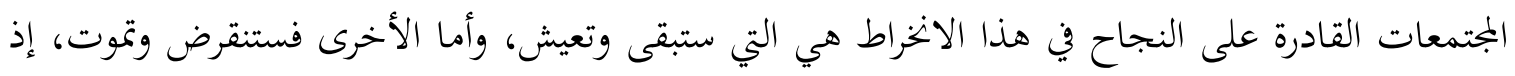
لا مكان تحت الشمس لمن لا يعرف كيف يتأقلم ويقدم شيئا ما لإنسانية الإنسان، ومِن تَمَّ فلا مستقبل للخطابات الأصولية المليئة بالاتحامات والغيظ والحقد على العالم الحديث واعتباره أصل الشر والبلاء. وتختم الدراسة الخامسة بالبحث في (التسامح واللاتسامح في التراث الإسلامي)، مبرزة أنه على عكس ما يقول المسلمون من أنه شهد التاريخ الإسلامي قمعا صريحا ودائما للحرية الدينية، ولكن أركون يعود ليبين أن الإسلام ليس هو وحده العامل المؤثر، وإنما هناك عوامل أخرى كالعقائد القديمة السابقة عليه إضافة إلى 
معطيات ديمغرافية واقتصادية وسياسية سائدة في تلك الفترة. وإذا وسعنا دائرة البحث لتشمل العصور الحديثة فإن الخطاب الإسلامي المهيمن منذ عام 1930م لا يزال يشكل عقبة كؤود أمام دخول المسلمين والعرب عصر التسامح الذي هو وجه من وجوه الحداثة الفكرية والعقلية بل إنه لا يزال مصرا على ذلك بنوع من الاستمرارية والتواصلية العنيدة والفعالية النفسية - الاجتماعية والسياسية التي لم تعد تحتمل، إننا نجد العقل الأصول المهيمن لا يزال مصرا على تدارك تأخرهم التاريخي واللحاق بركب الأمم المتحضرة، نجده يلجم هذا التطور ويوقفه عند حده. 